\title{
Management- und Führungsseminar für Oberärztinnen und Oberärzte
}

Management- und Führungsaufgaben sind zentrale oberärztliche Aufgaben. Anfänglich steht in der Oberarzttätigkeit wohl der fachliche Aspekt im Vordergrund, gilt es doch den medizinischen Klinikalltag zu meistern und weitere medizinische Fachkompetenz zu erlangen. In ihrer Tätigkeit werden Oberärztinnen und Oberärzte aber zunehmend auch mit Managementfragen konfrontiert. Führungskompetenz und soziale Kompetenz werden $\mathrm{zu}$ wichtigen Elementen beim Führen der eigenen Abteilungen.

Der richtige Einsatz von Managementtools entscheidet weitgehend über Erfolg oder Misserfolg in der täglichen Führungsarbeit. Darüber hinaus sind Kennen und Verstehen von ausgewählten Grundbegriffen des modernern Managements aber auch unabdingbare Voraussetzungen, um die weitere Zukunft erfolgreich zu gestalten. Dies gilt sowohl für eine zukünftige Karriere als Leitende(r) Ärztin/Arzt oder Chefärztin/Chefarzt wie auch für eine eigene Praxistätigkeit oder eine alternative Karriere. Management wird oft durch «learning by doing» und durch eigene Erfahrung - auch schmerzliche - erlernt. Um die eigenen Erfahrungen strukturiert zu überdenken und weiterzuentwickeln, wurde ein anspruchsvoller Kurs konzipiert, der konzis auf die wesentlichsten Fragen des Führungsalltags eingeht.

Der Kurs wird in Zusammenarbeit mit der FMH und dem VSAO angeboten. Eine Anrechnung durch einzelne Fachgesellschaften als persönliche Fortbildung ist möglich.

\section{Kursziel}

Ziel ist es, Oberärztinnen und Oberärzten konkrete Erkenntnisse und Tools zu vermitteln, damit sie ihre eigene Führungs- und Sozialkompetenz reflektieren, optimieren und - situationsgerecht - effizient einsetzen können. Dabei wird gezielt auf die Bedürfnisse der Teilnehmenden eingegangen, sei es im Dialog, in Übungen oder Simulationen. Themenbezogene Referate von Experten geben den theoretischen Hintergrund.

\section{Kursinhalt}

Der Kurs beinhaltet relevante Elemente des Führungs- und Managementalltags von Oberärztinnen und Oberärzten. Die Kursinhalte sind in Zusammenarbeit mit aktiven VSAO-Mitgliedern erarbeitet und auf das Praxisrelevante konzentriert worden.

- Führungsverständnis, Führungsstile und ihre Anwendung, Umgang mit Macht;

- Analyse und Entwicklung des eigenen Führungsstils, Umgang mit Kritik;

- Konfliktanalyse und Konfliktmanagement, Gesetzmässigkeiten und Interventionsmöglichkeiten, Vermeiden von Konflikten;

- Gesprächsführung: Mitarbeitergespräche, Qualifikationsgespräche, schwierige Gespräche (nicht Patientengespräche) usw.;

- Mitarbeiterentwicklung: Mitarbeiter fordern und fördern, Umgang mit schwierigen Mitarbeitern, Teambildung, Vereinbarungskultur;

- Selbstmanagement: persönliches Ressourcenund Zeitmanagement, Balance zwischen Arbeit, Karriere, Familie und Freizeit;

- Projektvorbereitung: alle Teilnehmenden erhalten die Möglichkeit, eine Thematik aus dem eigenen persönlichen Umfeld im Sinne eines kleinen Projekts weiterzubearbeiten;

- Projektanalyse und Besprechung mit den Kursteilnehmenden und den -verantwortlichen.

\section{Zielpublikum}

- Exklusiv Oberärztinnen und Oberärzte;

- mindestens 1 bis 2 Jahre Praxis in Oberarztfunktion;

- offen für alle medizinischen Fachgebiete.

\section{Dozentinnen und Dozenten}

Fachkompetente Experten mit eigener Praxiserfahrung und Verständnis für den Spitalalltag vermitteln die jeweiligen Kursinhalte theoretisch und praxisbezogen. 


\section{Informationen und Anmeldung}

\section{Kursdaten}

Donnerstag und Freitag 2./3. Dezember 2004

Freitag und Samstag 25./26. Februar 2005

Donnerstag-Samstag 7.-9. April 2005

Projektbesprechung September 2005

\section{Kursorte}

Olten und Bern, Tageskurse ohne Übernachtung.

\section{Kurskosten}

8 Kurstage inklusive Mittagessen, Pausenverpflegung, Infrastruktur, Kursunterlagen und Projektbetreuung: Fr. 4400.-.

\section{Anmeldeschluss: 15. Juli 2004}

\section{Kursverantwortliche}

- RA Dr. iur. Christoph Zenger, Bern;

- Dr. med. Jean Biaggi MHA, Muri b. Bern.

\section{Kurssekretariat}

zenger \& biaggi führungsseminare, Claudia Baeriswyl, Egghölzliweg 4a, Postfach 418, 3074 Muri, Tel. 03195173 20, Fax 03195172 10, E-Mail: jean.biaggi@bluewin.ch. 